Pacific Journal of Mathematics

DIAGONALIZING PROJECTIONS IN MULTIPLIER ALGEBRAS 


\section{DIAGONALIZING PROJECTIONS IN MULTIPLIER ALGEBRAS AND IN MATRICES OVER A $C^{*}$-ALGEBRA}

SHUANG ZHANG

Assume that $\mathscr{A}$ is a $C^{*}$-algebra with the FS property ([3] and [16]). We prove that every projection in $M_{n}(\mathscr{A}) \quad(n \geq 1)$ or in $L\left(\mathscr{H}_{\mathscr{A}}\right)$ is homotopic to a projection whose diagonal entries are projections of $\mathscr{A}$ and off-diagonal entries are zeros. This yields partial answers for Questions 7 and 8 raised by M. A. Rieffel in [18]. If $\mathscr{A}$ is $\sigma$-unital but non-unital, then every projection in the multiplier algebra $M(\mathscr{A})$ is unitarily equivalent to a diagonal projection, and homotopic to a block-diagonal projection with respect to an approximate identity of $\mathscr{A}$ consisting of an increasing sequence of projections. The unitary orbits of self-adjoint elements of $\mathscr{A}$ and $M(\mathscr{A})$ are also considered.

0. Introduction. It is well known that a projection in $M_{n}(\mathbb{C})$ or in $\mathbf{L}(\mathscr{H})$ is homotopic to a diagonal projection whose diagonal entries are either 1 or 0 , where $M_{n}(\mathbb{C})$ is the algebra consisting of $n \times n$ scalar matrices and $\mathbf{L}(\mathscr{H})$ is the algebra consisting of bounded operators on a separable Hilbert space $\mathscr{H}$. The following natural question comes up: if $\mathbb{C}$ is replaced by a $C^{*}$-algebra $\mathscr{A}$, is every projection in $M_{n}(\mathscr{A})$ or $L\left(\mathscr{H}_{\mathscr{A}}\right)$ homotopic to a diagonal projection whose diagonal entries are projections of $\mathscr{A}$ and off-diagonal entries are zeros? Here $M_{n}(\mathscr{A})$ is the $C^{*}$-algebra of $n \times n$ matrices over $\mathscr{A}$ and $L\left(\mathscr{H}_{\mathscr{A}}\right)$ can be regarded as bounded infinite matrices over $\mathscr{A}$ whose adjoints exist (see $\S 1$ for a more precise description). Certainly, diagonalizing projections of $M_{n}(\mathscr{A})$ for $n \geq 1$ would yield information about $K_{0}(\mathscr{A})$ (here diagonalizing projections in the sense of Murray-von Neumann is enough for this purpose).

Concerning the matrix algebra $M_{n}(\mathscr{A}), \mathrm{R}$. V. Kadison proved ([13] and [14]) that if $\mathscr{A}$ is a von Neumann algebra, then every normal element in $M_{n}(\mathscr{A})$ is unitarily equivalent to a diagonal normal matrix over $\mathscr{A}$. Consequently, every projection in $M_{n}(\mathscr{A})$ is homotopic to a diagonal projection, since the unitary group of a von Neumann algebra is connected. In general, we certainly do not expect a positive answer for the question if $\mathscr{A}$ is an arbitrary $C^{*}$-algebra. K. Grove and 
G. K. Pedersen have pointed out $([11,1.3])$ that if $\mathscr{A}$ is the algebra $C\left(S^{2}\right)$, the algebra of complex-valued continuous functions on $S^{2}$, then there exists a projection in $M_{2}(\mathscr{A})$ which is not unitarily equivalent to any diagonal projection. However, we do expect a positive answer for a large class of $C^{*}$-algebras.

The author has proved ([22]) that if $\mathscr{A}$ is a $C^{*}$-algebra with FS, then every projection in $M_{n}(\mathscr{A})$ or in $L\left(\mathscr{H}_{\mathscr{A}}\right)$ is Murray-von Neumann equivalent to a diagonal projection. In this note, we will strengthen the previous results to unitary equivalence or homotopy. We prove that if $\mathscr{A}$ is a $C^{*}$-algebra with FS (not necessarily $\sigma$-unital), and if $p$ is a projection of the multiplier algebra $M(\mathscr{A})$, then every projection $q$ of $\mathscr{A}$ is homotopic to a projection $q^{\prime}=p_{1}+p_{2}$, where $p_{1}$ is a projection of $p \mathscr{A} p$ and $p_{2}$ is a projection of $(1-p) \mathscr{A}(1-p)$. As a special case, by induction we conclude that every projection in $M_{n}(\mathscr{A})$ is homotopic to a diagonal projection. This yields partial answers for Questions 7 and 8 raised by M. A. Rieffel in [18]. If $\mathscr{A}$ is $\sigma$-unital and $\left\{e_{n}\right\}$ is a fixed sequence of mutually orthogonal projections of $\mathscr{A}$ such that $\sum_{n=1}^{\infty} e_{n}=1$, we prove that every projection in $M(\mathscr{A})$ is unitarily equivalent to a diagonal projection and homotopicto a block-diagonal projection with respect to the decomposition $\sum_{n=1}^{\infty} e_{n}=1$. As a consequence, every projection in $L\left(\mathscr{H}_{\mathscr{A}}\right)$ is unitarily equivalent (and hence homotopic) to a diagonal projection. In addition, the unitary orbits of self-adjoint elements of $\mathscr{A}$ or $M(\mathscr{A})$ are considered.

The class of $C^{*}$-algebras with FS includes many interesting subclasses of $C^{*}$-algebras. Obviously, AF algebras, the Calkin algebra, von Neumann algebras and $A W^{*}$-algebras have FS. The BunceDeddens algebras have FS ([2]). All purely infinite, simple $C^{*}$-algebras have FS ([24, Part I (1.3)] and [25]); in particular, the Cuntz algebras $\mathscr{O}_{n}$ and $\mathscr{O}_{A}$, where $2 \leq n \leq \infty$ and $A$ is an irreducible scalar matrix, have FS. Certain irrational rotation $C^{*}$-algebras have FS ([9]). Many corona and multiplier algebras have FS ([5], [24, Part I] and [24, Part IV]). L. G. Brown and G. K. Pedersen have recently proved ([5]) that a $C^{*}$-algebra $\mathscr{A}$ has FS if and only if $M_{n}(\mathscr{A})$ has FS for all $n \geq 1$; and $\mathscr{A}$ has FS if and only if $\mathscr{A}$ has real rank zero. In [21], [22], [23] and [24] the author has investigated the multiplier and corona algebras of $C^{*}$-algebras with FS from various angles.

1. Notations. If $\mathscr{A}$ is a $C^{*}$-algebra, we denote the Banach space double dual of $\mathscr{A}$ by $\mathscr{A}^{* *}$ and the multiplier algebra of $\mathscr{A}$ by $M(\mathscr{A})$; where $M(\mathscr{A})=\left\{m \in \mathscr{A}^{* *}: x m, m x \in \mathscr{A} \forall x \in \mathscr{A}\right\}$ ([1], [7], [15], among others). 
Let $\mathscr{H}_{\mathscr{A}}=\left\{\left\{a_{i}\right\}: a_{i} \in \mathscr{A}\right.$ and $\sum_{i=1}^{\infty} a_{i}^{*} a_{i}$ converges in norm $\}$. Then $\mathscr{H}_{\mathscr{A}}$ becomes a Hilbert $\mathscr{A}$-module with the $\mathscr{A}$-valued inner product

$$
\left\langle\left\{a_{i}\right\},\left\{b_{i}\right\}\right\rangle=\sum_{i=1}^{\infty} a_{i}^{*} b_{i} \quad \text { for all }\left\{a_{i}\right\},\left\{b_{i}\right\} \in \mathscr{H}_{\mathscr{A}} .
$$

We denote by $L\left(\mathscr{H}_{\mathscr{A}}\right)$ the set of all bounded module maps with an adjoint and by $K\left(\mathscr{H}_{\mathscr{A}}\right)$ a closed ideal of $L\left(\mathscr{H}_{\mathscr{A}}\right)$ called the "compact maps"; more precisely, $K\left(\mathscr{H}_{\mathscr{A}}\right)$ is the norm closure of the set of all "finite rank" module maps, $\left\{\sum_{i=1}^{n} \theta_{x_{i}, y_{i}}: x_{i}, y_{i} \in \mathscr{H}_{\mathscr{A}}\right.$ and $\left.n \in \mathbb{N}\right\}$. Here for any pair of elements $x$ and $y$ in $\mathscr{H}_{\mathscr{A}}, \theta_{x, y}$ is defined by $\theta_{x, y}(a)=x\langle y, a\rangle \in \mathscr{H}_{\mathscr{A}}$ for all $a \in \mathscr{H}_{\mathscr{A}}$ ([15]). It was proved ([15]) that

$$
L\left(\mathscr{H}_{\mathscr{A}}\right) \cong M(\mathscr{A} \otimes \mathscr{K}) \text { and } K\left(\mathscr{H}_{\mathscr{A}}\right) \cong \mathscr{A} \otimes \mathscr{K}
$$

as $C^{*}$-algebras, where $\mathscr{H}$ is the algebra consisting of compact operators on $\mathscr{H}$. The formulation of $L\left(\mathscr{H}_{\mathscr{A}}\right)$ and $K\left(\mathscr{H}_{\mathscr{A}}\right)$ are closely analogous to those of $\mathrm{L}(\mathscr{H})$ and $\mathscr{K}$.

If $\mathscr{A}$ is a unital $C^{*}$-algebra, we will denote the unitary group of $M_{n}(\mathscr{A})$ by $U_{n}(\mathscr{A})$ and the path component of $U_{n}(\mathscr{A})$ containing the identity by $U_{n}^{0}(\mathscr{A})$. In particular, we will denote $U_{1}^{0}(\mathscr{A})$ by $U_{0}(\mathscr{A})$.

If $p$ and $q$ are projections in $\mathscr{A}, p \sim q$ means that $p$ and $q$ are equivalent in the sense of Murray-von Neumann, and $p \approx q$ means that $p$ and $q$ are homotopic, i.e., in the same norm path component of projections in $\mathscr{A}$. It is well known that $p \approx q$ if and only if there exists a unitary element $v$ in $U_{0}(\mathscr{A})$ such that $v p v^{*}=q$. We denote the matrix units of $\mathscr{K}$ by $\left\{e_{i j}\right\}$.

2. Key Lemmas. The following technical lemmas are the key of this paper:

2.1. Lemma. Suppose that $\mathscr{A}$ is a $C^{*}$-algebra with FS (not necessarily $\sigma$-unital) and $p$ is a projection in $M(\mathscr{A})$. If $q$ is a projection in $\mathscr{A}$, then for any $\varepsilon_{0}>0$ there exists a projection $q^{\prime}$ in $\mathscr{A}$ such that both $p q^{\prime} p$ and $(1-p) q^{\prime}(1-p)$ have finite spectra and $\left\|q-q^{\prime}\right\|<\varepsilon_{0}$. More precisely, the projection $q^{\prime}$ has the following form:

$$
q^{\prime}=\left(\begin{array}{ccc}
f_{0} & 0 & 0 \\
0 & a_{0} & b_{0} \\
0 & b_{0}^{*} & c_{0}
\end{array}\right)
$$

where $f_{0}$ and the range of $a_{0}$ are mutually orthogonal subprojections of $p$. Consequently $q^{\prime} \approx q$ if $\varepsilon_{0}<1$. 
Proof. Let $q=\left(\begin{array}{cc}a & b \\ b^{*} & c\end{array}\right)$ be the decomposition of $q$ with respect to $p+(1-p)=1$. It follows that $a-a^{2}=b b^{*}, c-c^{2}=b^{*} b, a b+b c=b$, $0 \leq a \leq p$ and $0 \leq c \leq 1-p$. (Actually these conditions are also sufficient for $q$ to be a projection.) We will start with the idea in [6] and then go further to construct a projection $q^{\prime}=\left(\begin{array}{c}a^{\prime} b^{\prime} \\ b^{\prime *} \\ c^{\prime}\end{array}\right)$ such that both $\sigma\left(a^{\prime}\right)$ and $\sigma\left(c^{\prime}\right)$ are finite sets, and $q^{\prime}$ is close to $q$ in norm.

Let $0<\delta<1$ be a fixed positive number and $\varepsilon$ be another positive number such that $3 \varepsilon<\delta$. Since $\mathscr{A}$ has FS, there exists a positive element $c_{1}$ in $(1-p) \mathscr{A}(1-p)$ with a finite spectrum such that

$$
\left\|c-c_{1}\right\|<\varepsilon \text {. }
$$

Set $e=\chi_{(\delta, \infty)}\left(c_{1}-c_{1}^{2}\right)$. If $\delta_{1}$ is the smaller root of $t^{2}-t+\delta=0$, then $e=\chi_{\left(\delta_{1}, 1-\delta_{1}\right)}\left(c_{1}\right)$ which is a projection in $(1-p) \mathscr{A}(1-p)$.

Set $c_{0}=c_{1} e+\chi_{\left(1-\delta_{1}, 1\right]}\left(c_{1}\right)$. Then $\sigma\left(c_{0}\right)$ is a finite set, $c_{0}-c_{0}^{2}=$ $e\left(c_{1}-c_{1}^{2}\right) e \in e \mathscr{A} e$ and $\left\|c_{0}-c_{1}\right\| \leq \delta_{1}$. It follows that

$$
\left\|c_{0}-c\right\| \leq \varepsilon+\delta_{1}<\varepsilon+\sqrt{\delta} .
$$

Set $v=\left(e b^{*} b e\right)^{-1 / 2}\left(e b^{*}\right)$, of course where $\left(e b^{*} b e\right)^{-1}$ is taken in $e \mathscr{A} e$. Since $e\left(c_{1}-c_{1}^{2}\right) e \geq \delta e$ and hence $e b^{*} b e \geq(\delta-3 \varepsilon) e$, $\left(e b^{*} b e\right)^{-1 / 2}$ exists. It is clear that $v v^{*}=e$.

Set $b_{0}=v^{*}\left(c_{0}-c_{0}^{2}\right)^{1 / 2}$. Then $b_{0}^{*} b_{0}=c_{0}-c_{0}^{2}$.

Set $a_{0}=v^{*}\left(e-c_{0}\right) v$. Then $a_{0}-a_{0}^{2}=b_{0} b_{0}^{*}$ and $a_{0} b_{0}+b_{0} c_{0}=b_{0}$.

If we first fix $\delta$ small enough, then we choose $\varepsilon$ small enough and $c_{1}$ satisfying (1) such that $\left\|c-c_{0}\right\|,\left\|b-b_{0}\right\|$ and $\left\|\left(a-a^{2}\right)-\left(a_{0}-a_{0}^{2}\right)\right\|$ are all smaller than any preassigned positive number. However, $\left\|a-a_{0}\right\|$ can be equal to one. Here we give details for further reference.

It is obvious that

$$
\left\|b^{*} b-\left(c_{1}-c_{1}^{2}\right)\right\| \leq 3\left\|c-c_{1}\right\|<3 \varepsilon .
$$

Since $\left\|(1-e) b^{*} b(1-e)-(1-e)\left(c_{1}-c_{1}^{2}\right)(1-e)\right\| \leq 3 \varepsilon$ and $\left\|(1-e)\left(c_{1}-c_{1}^{2}\right)(1-e)\right\| \leq \delta$, it is easily seen that

$$
\|b(1-e)\| \leq \sqrt{3 \varepsilon+\delta} .
$$

Since $e b^{*} b e \geq(\delta-3 \varepsilon) e$, then

$$
\left\|\left(e b^{*} b e\right)^{-1}\right\| \leq(\delta-3 \varepsilon)^{-1} .
$$

By $[12,126]$ and (3), we can choose $\varepsilon$ small enough such that

$$
\left\|\left(e b^{*} b e\right)^{1 / 2}-\left[e\left(c_{1}-c_{2}^{2}\right) e\right]^{1 / 2}\right\|<\delta .
$$


By (4) and (6) we can choose $\varepsilon$ small enough such that

$$
\begin{aligned}
\left\|b_{0}-b\right\| & \leq\left\|v^{*}\left(c_{0}-c_{0}^{2}\right)^{1 / 2}-v^{*}\left(e b^{*} b e\right)^{1 / 2}\right\|+\|b(1-e)\| \\
& \leq\left\|\left[e\left(c_{1}-c_{1}^{2}\right) e\right]^{1 / 2}-\left(e b^{*} b e\right)^{1 / 2}\right\|+\sqrt{3 \varepsilon+\delta} \\
& <\delta+\sqrt{3 \varepsilon+\delta}
\end{aligned}
$$

Consequently,

$$
\begin{aligned}
\left\|\left(a-a^{2}\right)-\left(a_{0}-a_{0}^{2}\right)\right\| & =\left\|b b^{*}-b_{0} b_{0}^{*}\right\| \\
& \leq 2\left\|b_{0}-b\right\|<2 \delta+2 \sqrt{3 \varepsilon+\delta} .
\end{aligned}
$$

It is clear from construction that $q_{0}=\left(\begin{array}{ll}a_{0} & b_{0} \\ b_{0}^{*} & c_{0}\end{array}\right)$ is a projection. By Lemma (2.4) of [21], $\sigma\left(a_{0}\right) \backslash\{0,1\}=\sigma\left(1-c_{0}\right) \backslash\{0,1\}$, and hence $\sigma\left(a_{0}\right)$ is also a finite set. The idea of constructing the projection $q_{0}$ is due L. G. Brown ([6]) for different purpose.

We will go further to adjust $q_{0}$ to a projection $q^{\prime}=\left(\begin{array}{cc}a^{\prime} & b^{\prime} \\ b^{\prime *} & c^{\prime}\end{array}\right)$ so that $\left\|a-a^{\prime}\right\|$ is small, too. Set $f=v^{*} v$. Then $f$ is a subprojection of $p$ and $f a_{0}=a_{0} f=a_{0}$. We claim that $\left\|f a f-a_{0}\right\|$ can be arbitrarily small if $\delta, \varepsilon$ and $c_{1}$ are properly chosen. To prove this claim, we need the following estimates.

$$
\begin{aligned}
\left\|e\left(b^{*} b\right)^{1 / 2}(1-e)\right\| & =\left\|e\left[\left(b^{*} b\right)^{1 / 2}-\left(c_{1}-c_{1}^{2}\right)^{1 / 2}\right](1-e)\right\| \\
& \leq\left\|\left(b^{*} b\right)^{1 / 2}-\left(c_{1}-c_{1}^{2}\right)^{1 / 2}\right\| .
\end{aligned}
$$

Then by $[12,126]$ and

$$
\left[\left(e b^{*} b e\right)^{1 / 2}\right]^{2}=e b^{*} b e=\left[e\left(b^{*} b\right)^{1 / 2} e\right]^{2}+e\left(b^{*} b\right)^{1 / 2}(1-e)\left(b^{*} b\right)^{1 / 2} e,
$$

for a fixed $\delta>0$ we can choose $\varepsilon$ small enough (by (3)) such that

$$
\begin{gathered}
\left\|\left(b^{*} b\right)^{1 / 2}-\left(c_{1}-c_{1}^{2}\right)^{1 / 2}\right\|<\frac{\delta^{2}}{2} \text { and } \\
\left\|\left(e b^{*} b e\right)^{1 / 2}-e\left(b^{*} b\right)^{1 / 2} e\right\|<\delta \sqrt{\frac{\delta}{2}}
\end{gathered}
$$

Since

$$
\begin{aligned}
f\left(a-a_{0}\right) f & =v^{*} e v\left(a-a_{0}\right) v^{*} e v \\
& =v^{*} e\left(v a v^{*}-v a_{0} v^{*}\right) e v \\
& =v^{*}\left[e c_{0} e-v(p-a) v^{*}\right] v
\end{aligned}
$$

then

$$
\begin{aligned}
\left\|f\left(a-a_{0}\right) f\right\| & \leq\left\|e c_{0} e-e c e\right\|+\left\|e c e-v(p-a) v^{*}\right\| \\
& <\varepsilon+\left\|e c e-v(p-a) v^{*}\right\| .
\end{aligned}
$$


Since $(1-a) b=b c, p(1-a) b=b p(c)$ for any polynomial $p(t)$. Approximating by polynomials, we obtain that $\sqrt{1-a} b=b \sqrt{c}$, and hence

$$
b^{*}(1-a) b=c^{2}-c^{3}=\left(b^{*} b\right)^{1 / 2} c\left(b^{*} b\right)^{1 / 2} .
$$

It follows that

$$
\begin{aligned}
v(p-a) v^{*} & =\left(e b^{*} b e\right)^{-1 / 2} e b^{*}(p-a) b e\left(e b^{*} b e\right)^{-1 / 2} \\
& =\left(e b^{*} b e\right)^{-1 / 2} e\left[b^{*} b-b^{*} a b\right] e\left(e b^{*} b e\right)^{-1 / 2} \\
& =\left(e b^{*} b e\right)^{-1 / 2}\left[e\left(b^{*} b\right)^{1 / 2} c\left(b^{*} b\right)^{1 / 2} e\right]\left(e b^{*} b e\right)^{-1 / 2} \\
& =\left(e b^{*} b e\right)^{-1 / 2}\left[h_{1}+h_{2}\right]\left(e b^{*} b e\right)^{-1 / 2}
\end{aligned}
$$

where

$$
\begin{aligned}
h_{1}= & e\left(b^{*} b\right)^{1 / 2} e c e\left(b^{*} b\right)^{1 / 2} e \\
= & \left(e b^{*} b e\right)^{1 / 2} c\left(e b^{*} b e\right)^{1 / 2}+\left[e\left(b^{*} b\right)^{1 / 2} e-\left(e b^{*} b e\right)^{1 / 2}\right] c\left(e b^{*} b e\right)^{1 / 2} \\
& +\left(e b^{*} b e\right)^{1 / 2} c\left[e\left(b^{*} b\right)^{1 / 2} e-\left(e b^{*} b e\right)^{1 / 2}\right] \\
& +\left[e\left(b^{*} b\right)^{1 / 2} e-\left(e b^{*} b e\right)^{1 / 2}\right] c\left[e\left(b^{*} b\right)^{1 / 2} e-\left(e b^{*} b e\right)^{1 / 2}\right], \\
h_{2}= & e\left(b^{*} b\right)^{1 / 2}(1-e) c e\left(b^{*} b\right)^{1 / 2} e \\
& +e\left(b^{*} b\right)^{1 / 2} e c(1-e)\left(b^{*} b\right)^{1 / 2} e \\
& +e\left(b^{*} b\right)^{1 / 2}(1-e) c(1-e)\left(b^{*} b\right)^{1 / 2} e .
\end{aligned}
$$

If $\delta$ is first fixed small enough, and $\varepsilon$ and $c_{1}$ can be chosen such that $6 \varepsilon<\delta$ and

$$
\begin{aligned}
& \left\|\left(e b^{*} b e\right)^{-1 / 2} h_{1}\left(e b^{*} b e\right)^{-1 / 2}-e c e\right\| \\
& \leq 2\left\|\left(e b^{*} b e\right)^{-1 / 2}\right\|\left\|e\left(b^{*} b\right)^{1 / 2} e-\left(e b^{*} b e\right)^{1 / 2}\right\|\|c\| \\
& \quad+\left\|\left(e b^{*} b e\right)^{-1 / 2}\right\|^{2}\left\|e\left(b^{*} b\right)^{1 / 2} e-\left(e b^{*} b e\right)^{1 / 2}\right\|^{2}\|c\| \\
& \leq 2 \frac{\delta \sqrt{\delta / 2}}{\sqrt{\delta-3 \varepsilon}}+\left[\frac{\delta \sqrt{\delta / 2}}{\sqrt{\delta-3 \varepsilon}}\right]^{2}<\delta^{2}+2 \delta,
\end{aligned}
$$

(where using (5), (10) and (11)) and

$$
\begin{aligned}
& \left\|\left(e b^{*} b e\right)^{-1 / 2} h_{2}\left(e b^{*} b e\right)^{-1 / 2}\right\| \\
& \leq 2\left\|\left(e b^{*} b e\right)^{-1 / 2}\right\|^{2}\left\|e\left(b^{*} b\right)^{1 / 2}(1-e)\right\|\|c\|\left\|\left(b^{*} b\right)^{1 / 2}\right\| \\
& \quad+\left\|\left(e b^{*} b e\right)^{-1 / 2}\right\|^{2}\left\|e\left(b^{*} b\right)^{1 / 2}(1-e)\right\|^{2}\|c\| \\
& <(\delta-3 \varepsilon)^{-1}\left[\delta^{2}+\frac{\delta^{4}}{4}\right]<2 \delta+\delta^{2},
\end{aligned}
$$

where we used $\delta-3 \varepsilon>\delta / 2$. Consequently,

$$
\begin{gathered}
\left\|v(p-a) v^{*}-e c e\right\| \leq 4 \delta+2 \delta^{2}, \quad \text { and so } \\
\left\|f\left(a-a_{0}\right) f\right\|<\varepsilon+4 \delta+2 \delta^{2} \quad \text { by (12). }
\end{gathered}
$$


If $\delta$ is fixed small enough and $\varepsilon$ is chosen small enough, then $\| f a f-$ $a_{0} \|$ can be arbitrarily small if $c_{1}$ satisfies (1).

Moreover, by properly choosing $\delta>0, \varepsilon$ and $c_{1}$ in a similar way we can require that $\|(p-f) a f\|$ is less than any preassigned positive number. This can be done as follows.

Since $a-a^{2}=b b^{*}$ and the spectral mapping theorem, it is clear $\|b\| \leq 1 / 2$. Since $(1-a) b=b c$, we have

$$
\begin{aligned}
-(1-f) a v^{*}= & (1-f)(1-a) b e\left(e b^{*} b e\right)^{-1 / 2} \\
= & b c e\left(e b^{*} b e\right)^{-1 / 2}-b e\left(e b^{*} b e\right)^{-1} e b^{*} b c e\left(e b^{*} b e\right)^{-1 / 2} \\
= & b c e\left(e b^{*} b e\right)^{-1 / 2}-b e\left(e b^{*} b e\right)^{-1} e b^{*} b e c e\left(e b^{*} b e\right)^{-1 / 2} \\
& -b e\left(e b^{*} b e\right)^{-1} e b^{*} b(1-e) c e\left(e b^{*} b e\right)^{-1 / 2} \\
= & b(1-e) c e\left(e b^{*} b e\right)^{-1 / 2} \\
& -b e\left(e b^{*} b e\right)^{-1} e b^{*} b(1-e) c e\left(e b^{*} b e\right)^{-1 / 2} .
\end{aligned}
$$

It follows that

$$
\begin{aligned}
\|(1- & f) a f\|\leq\|(1-f) a v^{*} \| \\
\leq & \|b\|\|(1-e) c e\|\left\|\left(e b^{*} b e\right)^{-1 / 2}\right\| \\
& +\|b\|\left\|\left(e b^{*} b e\right)^{-1}\right\|\left\|e\left(b^{*} b\right)(1-e)\right\|\|c\|\left\|\left(e b^{*} b e\right)^{-1 / 2}\right\| \\
& <\frac{\varepsilon}{2}\left[\frac{1}{\sqrt{\delta-3 \varepsilon}}\right]+\frac{1}{2}\left[\frac{1}{\delta-3 \varepsilon}\right](3 \varepsilon)\left[\frac{1}{\sqrt{\delta-3 \varepsilon}}\right] \\
& <\left[\frac{\varepsilon}{2}\right] \sqrt{\frac{2}{\delta}}+\left[\frac{3 \varepsilon}{2}\right]\left[\frac{2}{\delta}\right]^{3 / 2}
\end{aligned}
$$

where we use (1), (3), (5) and the facts:

$$
\begin{gathered}
\|(1-e) c e\|=\left\|(1-e)\left(c-c_{1}\right) e\right\| \leq\left\|c-c_{1}\right\|, \quad \text { and } \\
\left\|e b^{*} b(1-e)\right\|=\left\|e\left[b^{*} b-\left(c_{1}-c_{1}^{2}\right)\right](1-e)\right\| \leq\left\|b^{*} b-\left(c_{1}-c_{1}^{2}\right)\right\| .
\end{gathered}
$$

As a consequence of the last estimate and (8), for any $0<\lambda<1 / 2$, we can fix $\delta$ small enough and then choose $\varepsilon$ small enough such that $\sigma((p-f) a(p-f)) \subset[0, \lambda] \cup[1-\lambda, 1]$. This is because of the following estimates:

$$
\begin{aligned}
& (p-f)\left[\left(a-a^{2}\right)-\left(a_{0}-a_{0}^{2}\right)\right](p-f)=(p-f)\left(a-a^{2}\right)(p-f) \\
& =(p-f) a(p-f)-[(p-f) a(p-f)]^{2}-(p-f) a f a(p-f), \\
& \left\|(p-f) a(p-f)-[(p-f) a(p-f)]^{2}\right\| \\
& \quad \leq\left\|(p-f)\left[\left(a-a^{2}\right)-\left(a_{0}-a_{0}^{2}\right)\right](p-f)\right\|+\|(1-f) a f\|^{2} \\
& \quad \leq\left\|\left(a-a^{2}\right)-\left(a_{0}-a_{0}^{2}\right)\right\|+\|(p-f) a f\|^{2} .
\end{aligned}
$$


Set $f_{0}=\chi_{[1 / 2,1]}((p-f) a(p-f))$. Then $f_{0}$ is a projection in $(p-f) \mathscr{A}(p-f)$ such that $f_{0} a_{0}=a_{0} f_{0}=0$ and $\left\|f_{0}-(p-f) a(p-f)\right\| \leq$ $\lambda$. Set $a^{\prime}=a_{0}+f_{0}, b^{\prime}=b_{0}$ and $c^{\prime}=c_{0}$. Then $q^{\prime}=\left(\begin{array}{c}a^{\prime} \\ b^{\prime *} \\ c^{\prime}\end{array}\right)$ is a projection in $\mathscr{A}$ such that

$$
\text { (16) } \begin{aligned}
\left\|q^{\prime}-q\right\| \leq & \left\|\left(f_{0}+a_{0}\right)-a\right\|+2\left\|b_{0}-b\right\|+\left\|c_{0}-c\right\| \\
\leq & \left\|f\left(a-a_{0}\right) f\right\|+2\|f a(p-f)\| \\
& +\left\|f_{0}-(p-f) a(p-f)\right\|+2\left\|b_{0}-b\right\|+\left\|c_{0}-c\right\| .
\end{aligned}
$$

Combining all above estimates, we first fix $\lambda$ small enough, then fix $\delta$ small enough, and then choose $\varepsilon$ small enough and $c_{1}$ satisfying (1) so that each term on the right-hand side of (16) is small. Then $\left\|q-q^{\prime}\right\|$ is small. It is clear that $\sigma\left(p q^{\prime} p\right)=\sigma\left(f_{0}+a_{0}\right)$ is a finite set. The last sentence in the statement of this lemma is well known.

2.2. LemmA. Suppose that $\mathscr{A}$ is a $C^{*}$-algebra (not necessarily $\sigma$ unital) and $p$ is a projection in $M(\mathscr{A})$. If $q$ is a projection in $\mathscr{A}$ such that $\sigma(p q p) \neq[0,1]$, then there exist two projections $q_{1}$ and $q_{2}$ in $\mathscr{A}$ such that $q_{1} \leq p, q_{2} \leq 1-p$ and $q \approx q_{1}+q_{2}$.

Proof. Let $q=\left(\begin{array}{cc}a & b \\ b^{*} & c\end{array}\right)$ be the composition of $q$ with respect to $p+(1-p)=1$. Then $a=p q p, c=(1-p) q(1-p)$ and $b=p q(1-p)$. By [21, 2.4], $\sigma(a) \backslash\{0,1\}=\sigma(1-c) \backslash\{0,1\}$.

If $b=0$, then $q_{1}=a$ and $q_{2}=c$ are as desired. Assume that $b \neq 0$. If $1 \notin \sigma(c)$, then $\|c\|<1$. By the argument of $[8,1]$, $q$ is path connected to a subprojection $q_{1}$ of $p$. We can assume that $1 \in \sigma(c)$. Since $\sigma(c) \neq[0,1]$ and 0 is always in $\sigma(c)$, there is a $\lambda$ in $(0,1) \backslash \sigma(c)$. Then there exists a positive number $\varepsilon$ such that $(\lambda-\varepsilon, \lambda+\varepsilon) \cap \sigma(c)=\varnothing$. Since $b \neq 0$, we can assume that $\sigma(c) \cap(\lambda+\varepsilon, 1) \neq \varnothing$ (Otherwise, $\sigma(a) \cap(\lambda+\varepsilon, 1) \neq \varnothing$, we consider $a$ instead.) We will use a variation of $[8,1]$ to construct a path of projections for our purpose.

Define a family of continuous positive functions $\left\{f_{t}\right\}_{t \in[0,1]}$ from $[0,1]$ to $[0,1]$ with the following properties:

(1) $\lim _{t \rightarrow t_{0}}\left\|f_{t}-f_{t_{0}}\right\|_{\infty}=0$ for any $t_{0}$ in $[0,1]$;

(2) $f_{1}(s)=s$ for all $s$ in $[0,1]$;

$$
f_{0}(s)= \begin{cases}1, & \text { if } \lambda \leq s \leq 1, \\ \text { linear, } & \text { if } \lambda-\varepsilon<s<\lambda \\ 0, & \text { if } 0 \leq s \leq \lambda-\varepsilon\end{cases}
$$


(4) For all $t$ in $(0,1), f_{t}(s) \leq s$ if $s \in[0, \lambda-\varepsilon]$ and $f_{t}(s) \geq s$ if $s \in[\lambda, 1]$.

Since $q$ is a projection, $b c=(1-a) b$. Approximating by polynomials, we obtain that $b g(c)=g(1-a) b$ for any continuous function $g$ on $[0,1]$. Set

$$
\begin{aligned}
& c_{t}=f_{t}(c), \\
& b_{t}=b\left[\frac{f_{t}(c)-f_{t}(c)^{2}}{c-c^{2}}\right]^{1 / 2}, \\
& a_{t}=p-f_{t}(p-a) .
\end{aligned}
$$

Then $b_{t}$ and $c_{t}$ are well defined elements in $\mathscr{A}$ by the properties of $f_{t}$. Although $p-a$ is not in $p \mathscr{A} p$ if $p$ is in $M(\mathscr{A}) \backslash \mathscr{A}, p-f_{t}(p-a)$ is in $p \mathscr{A} p$ for $t \in[0,1]$. To see this, first, $f_{t}(p-a)$ is well defined for each $t \in[0,1]$ since $\sigma(p-a) \backslash\{0,1\}=\sigma(c) \backslash\{0,1\}$. Second, if we denote by $\pi$ the canonical map from $(p \mathscr{A} p)^{+}$to $(p \mathscr{A} p)^{+} / p \mathscr{A} p$, where $(p \mathscr{A} p)^{+}$is the $C^{*}$-algebra obtained by joining an identity to $p \mathscr{A} p$, then $p-f_{t}(p-a) \in p \mathscr{A} p$, since $\pi\left(p-f_{t}(p-a)\right)=\pi(p)-$ $f_{t}(\pi(p))=0$. It is easily verified that

$$
\begin{aligned}
a_{t}-a_{t}^{2} & =b_{t} b_{t}^{*}, \\
a_{t} b_{t} & =b_{t}\left(1-c_{t}\right), \\
c_{t}-c_{t}^{2} & =b_{t}^{*} b_{t} .
\end{aligned}
$$

Thus $q(t)=\left(\begin{array}{ll}a_{t} & b_{t} \\ b_{t}^{*} & c_{t}\end{array}\right)$ is a projection in $\mathscr{A}$ for each $t$ in $[0,1]$. By the property $(1)$ of $\left\{f_{t}\right\},\{q(t)\}_{t \in[0,1]}$ is contained in the same path component of projections in $\mathscr{A}$. Then $q(0) \approx q(1)=q$. Since $(\lambda-\varepsilon, \lambda) \cap \sigma(c)=\varnothing, c_{0}=f_{0}(c)=\chi_{[\lambda, 1]}(c)$ is a projection of $(1-p) \mathscr{A}(1-p)$. It is obvious that

$$
q(0)=\left(\begin{array}{ll}
a_{0} & b_{0} \\
b_{0}^{*} & c_{0}
\end{array}\right)=\left(\begin{array}{cc}
a_{0} & 0 \\
0 & c_{0}
\end{array}\right) .
$$

Consequently, $a_{0}$ is a projection of $p \mathscr{A} p$. Set $q_{1}=a_{0}$ and $q_{2}=c_{0}$, as desired.

Roughly speaking, with respect to a fixed sequential increasing approximate identity of $\mathscr{A}$ a block-diagonal projection of $M(\mathscr{A})$ whose blocks are with the same size is homotopic to a diagonal projection. More precisely, we have the following lemma:

2.3. Lemma. Suppose that $\mathscr{A}$ is a $\sigma$-unital, non-unital $C^{*}$-algebra with $F S$ and $\sum_{i=1}^{\infty}\left(s_{i 1}+s_{i 2}+\cdots+s_{i n}\right)=1$, where $\left\{s_{i j}: i \geq 1,1 \leq j \leq\right.$ $n$ \} are mutually orthogonal projections in $\mathscr{A}$ and the sum converges 
in the strict topology. If $p$ is a projection in $M(\mathscr{A})$ with the form $\sum_{i=1}^{\infty} p_{i}$, where $p_{i}$ is a projection in $\left(s_{i 1}+s_{i 2}+\cdots+s_{i n}\right) \mathscr{A}\left(s_{i 1}+s_{i 2}+\right.$ $\left.\cdots+s_{i n}\right)$ for $i \geq 1$, then $p \approx \sum_{i=1}^{\infty}\left(p_{i 1}+p_{i 2}+\cdots+p_{i n}\right)$, where $p_{i j}$ is a projection in $s_{i j} \mathscr{A} s_{i j}$ for $i \geq 1$ and $1 \leq j \leq n$.

Proof. It suffices to prove the case if $n=2$. If $n>2$, we simply employ the same proof recursively $n-1$ times by induction to reach the conclusion.

We write

$$
p_{i}=\left(\begin{array}{ll}
a_{i}^{*} & b_{i} \\
b_{i}^{*} & c_{i}
\end{array}\right)
$$

with respect to $s_{i 1}+s_{i 2}$. By Lemma (2.1), for each $i \geq 1$ we can find a projection

$$
p_{i}^{\prime}=\left(\begin{array}{ccc}
f_{i} & 0 & 0 \\
0 & a_{i}^{\prime} & b_{i}^{\prime} \\
0 & b_{i}^{\prime *} & c_{i}^{\prime}
\end{array}\right)
$$

in $\left(s_{i 1}+s_{i 2}\right) \mathscr{A}\left(s_{i 1}+s_{i 2}\right)$ such that $\left\|p_{i}^{\prime}-p_{i}\right\|<1 / 4$, and both $a_{i}^{\prime}$ and $c_{i}^{\prime}$ have finite spectra. Here we use the proof of Lemma (2.1) to properly choose a positive number $\delta_{i}$ and a positive element $c_{1 i}^{\prime}$ in $s_{i 2} \mathscr{A} s_{i 2}$ with a finite spectrum, then we have that

$$
\begin{gathered}
e_{i}=\chi_{\left(\delta_{i}, 1-\delta_{i}\right)}\left(c_{1 i}^{\prime}\right), \quad c_{i}^{\prime}=c_{1 i}^{\prime} e_{i}+\chi_{\left(1-\delta_{i}, 1\right)}\left(c_{1 i}^{\prime}\right), \\
v_{i}=\left(e_{i} b_{i}^{*} b_{i} e_{i}\right)^{-1 / 2}\left(e_{i} b_{i}^{*}\right), \quad b_{i}^{\prime}=v_{i}^{*}\left(c_{i}^{\prime}-c_{i i}^{\prime 2}\right)^{1 / 2}, \\
a_{i}^{\prime}=v_{i}^{*}\left(e_{i}-c_{1 i}^{\prime}\right) v_{i}
\end{gathered}
$$

and $f_{i}$ is a projection of $s_{i 1} \mathscr{A} s_{i 1}$ orthogonal to the range projection of $a_{i}^{\prime}$.

Let $p^{\prime}=\sum_{i=1}^{\infty} p_{i}^{\prime}$. Then $\left\|p^{\prime}-p\right\|<1 / 4$, and hence $p \approx p^{\prime}$.

Let $\sigma\left(c_{i}^{\prime}\right)=\left\{\lambda_{i 1}, \lambda_{i 2}, \cdots, \lambda_{i l_{l}}\right\}$ for each $i \geq 1$. It follows from the construction or $[21,2.4]$ that $\sigma\left(a_{i}^{\prime}\right)=\left\{1-\lambda_{i 1}, 1-\lambda_{i 2}, \ldots, 1-\lambda_{i l}\right\}$. We can write $c_{i}^{\prime}=\sum_{j=1}^{l_{t}} \lambda_{i j} r_{i j}$, where $\left\{r_{i j}: 1 \leq j \leq l_{i}\right\}$ is a set of mutually orthogonal projections in $s_{i 2} \mathscr{A} s_{i 2}$. Let $\lambda$ be any number in the open interval $\left(\frac{1}{2}, \frac{3}{4}\right)$ but not in $\bigcup_{i=1}^{\infty} \sigma\left(c_{i}^{\prime}\right)$. Let $\varepsilon=\min \left\{\lambda-\frac{1}{2}, \frac{3}{4}-\lambda\right\}$. For $i \geq 1$, if $\lambda_{i j}$ is in the open interval $(\lambda-\varepsilon, \lambda)$, we replace $\lambda_{i j}$ by $\lambda_{i j}^{\prime}=\lambda-\varepsilon$, and if $\lambda_{i j}$ is in $(\lambda, \lambda+\varepsilon)$, we replace $\lambda_{i j}$ by $\lambda_{i j}^{\prime}=\lambda+\varepsilon$. If $\lambda_{i j}$ is not in $(\lambda-\varepsilon, \lambda+\varepsilon)$, then we let $\lambda_{i j}^{\prime}=\lambda_{i j}$. Set $c_{i}^{\prime \prime}=\sum_{j=1}^{l_{t}} \lambda_{i j}^{\prime} r_{i j}$ for $i \geq 1$, and correspondingly set $b_{i}^{\prime \prime}=v_{i}^{*}\left(c_{i}^{\prime \prime}-c_{i}^{\prime \prime 2}\right)^{1 / 2}$ and $a_{i}^{\prime \prime}=$ $v_{i}^{*}\left(e_{i}-c_{i}^{\prime \prime}\right) v_{i}$. Then

$$
\begin{gathered}
\left\|a_{i}^{\prime}-a_{i}^{\prime \prime}\right\| \leq\left\|c_{i}^{\prime}-c_{i}^{\prime \prime}\right\|<\varepsilon \quad \text { and } \\
\left\|b_{i}^{\prime}-b_{i}^{\prime \prime}\right\| \leq\left\|\left(c_{i}^{\prime}-c_{i}^{\prime 2}\right)^{1 / 2}-\left(c_{i}^{\prime \prime}-c_{i}^{\prime \prime 2}\right)^{1 / 2}\right\|<\frac{1}{8} .
\end{gathered}
$$


It follows that

$$
p_{i}^{\prime \prime}=\left(\begin{array}{ccc}
f_{i} & 0 & 0 \\
0 & a_{i}^{\prime \prime} & b_{i}^{\prime \prime} \\
0 & b_{i}^{\prime \prime *} & c_{i}^{\prime \prime}
\end{array}\right)
$$

is a projection in $\left(s_{i 1}+s_{i 2}\right) \mathscr{A}\left(s_{i 1}+s_{i 2}\right)$ such that $\left\|p_{i}^{\prime}-p_{i}^{\prime \prime}\right\| \leq 2 \varepsilon+\frac{1}{4}<1$. Define $p^{\prime \prime}=\sum_{i=1}^{\infty} p_{i}^{\prime \prime}$. Then $\left\|p^{\prime}-p^{\prime \prime}\right\|<1$, and hence $p^{\prime} \approx p^{\prime \prime}$. The remaining job is to prove that $p^{\prime \prime}$ is homotopic to a desired diagonal projection.

Let $\left\{f_{t}\right\}_{t \in[0,1]}$ be the family of continuous functions defined in the proof of Lemma (2.2). Since $\sigma\left(c_{i}^{\prime \prime}\right)$ does not intersect with the open interval $(\lambda-\varepsilon, \lambda+\varepsilon)$ for $i \geq 1$, we can define

$$
\begin{aligned}
& c_{i}(t)=f_{t}\left(c_{i}^{\prime \prime}\right), \\
& b_{i}(t)=b_{i}^{\prime \prime}\left[\frac{f_{t}\left(c_{i}^{\prime \prime}\right)-f_{t}\left(c_{i}^{\prime \prime}\right)^{2}}{c_{i}^{\prime \prime}-c_{i}^{\prime \prime 2}}\right]^{1 / 2}, \\
& a_{i}(t)=p-f_{t}\left(p-a_{i}^{\prime \prime}-f_{i}\right) .
\end{aligned}
$$

Then $a_{i}(t), b_{i}(t)$ and $c_{i}(t)$ are well defined elements in $\left(s_{i 1}+s_{i 2}\right) \mathscr{A}$ $\left(s_{i 1}+s_{i 2}\right)$ for each $t$ in $[0,1]$ and $i \geq 1$ by the properties of $f_{t}$. Thus for each $t$ in $[0,1]$

$$
p_{i}(t)=\left(\begin{array}{cc}
a_{i}(t) & b_{i}(t) \\
b_{i}(t)^{*} & c_{i}(t)
\end{array}\right)
$$

is a projection in $\left(s_{i 1}+s_{i 2}\right) \mathscr{A}\left(s_{i 1}+s_{i 2}\right)$. It is easily seen that

$$
p_{i}(1)=p_{i}^{\prime \prime} \text { and } p_{i}(0)=\left(\begin{array}{cc}
a_{i}(0) & 0 \\
0 & c_{i}(0)
\end{array}\right) \text {, }
$$

where $a_{i}(0)$ is a projection of $s_{i 1} \mathscr{A} s_{i 1}$ and $c_{i}(0)$ is a projection of $s_{i 2} \mathscr{A} s_{i 2}$. Define $p(t)=\sum_{i=1}^{\infty} p_{i}(t)$ for each $t$ in $[0,1]$. Then $\{p(t)\}_{t \in[0,1]}$ is a path of projection in $M(\mathscr{A})$. It is obvious that

$$
p(1)=p^{\prime \prime} \text { and } p(0)=\sum_{i=1}^{\infty}\left(\begin{array}{cc}
a_{i}(0) & 0 \\
0 & c_{i}(0)
\end{array}\right) .
$$

Since the choice of $\left\{f_{t}\right\}_{t \in[0,1]}$ does not depend on $i$, the path $\{p(t)$ : $t \in[0,1]\}$ is continuous in the norm topology.

Set $p_{i 1}=a_{1}(0), p_{i 2}=c_{i}(0)$ for $i \geq 1$. Then

$$
p \approx p^{\prime} \approx p^{\prime \prime} \approx p(0)=\sum_{i=1}^{\infty}\left(p_{i 1}+p_{i 2}\right), \quad \text { as desired. }
$$

3. Diagonalizing projections in $\mathscr{A}$ and in $M_{n}(\mathscr{A})$. Since we will frequently employ the following well-known fact in this paper, we state it as a lemma. 
3.1. LeMMA. If $\mathscr{A}$ is a $C^{*}$-algebra, and if $p$ and $q$ are two $m u$ tually orthogonal projections in $\mathscr{A}$, then $p \sim q$ if and only if $p \approx q$.

Proof. Let $v$ be a partial isometry in $\mathscr{A}$ such that $v v^{*}=p$ and $v^{*} v=q$. Define $w=v+v^{*}+(1-p-q)$. Then $w$ is a selfadjoint unitary in $M(\mathscr{A})$ such that $w^{*} p w=q$. It is well known that $w \in U_{0}(\mathscr{A})$. It follows that $p \approx q$.

3.2. Theorem. Suppose that $\mathscr{A}$ is a $C^{*}$-algebra with FS and $p_{1}$, $p_{2}, \ldots, p_{n} \quad(n \geq 1)$ are mutually orthogonal projections in $M(\mathscr{A})$ such that $\sum_{i=1}^{n} p_{i}=1$. If $p$ is a projection in $\mathscr{A}$, then $p \approx \sum_{i=1}^{n} q_{i}$, where $q_{i}$ is a projection in $\mathscr{A}$ such that $q_{i} \leq p_{i}$ for $1 \leq i \leq n$.

Proof. Recursively using Lemma (2.1) and Lemma (2.2), we reach the conclusion.

The following theorem can be regarded as an analogue of the wellknown fact: Every projection in $M_{n}(\mathbb{C})$ is homotopic to a diagonal projection whose entries are either 1 or 0 .

3.3. TheOREM. Assume that $\mathscr{A}$ is a $C^{*}$-algebra with FS and $n \geq$ 1. If $p$ is a projection in $M_{n}(\mathscr{A})$, then $p \approx \sum_{i=1}^{n} p_{i} \otimes e_{i i}$, where $\left\{p_{i}\right\}$ is a set of projections in $\mathscr{A}$ such that

$$
p_{1} \leq p_{2} \leq \cdots \leq p_{n-1} \leq p_{n} .
$$

Proof. It has been recently proved ([5]) that $\mathscr{A} \otimes \mathscr{K}$ has FS if and only if $\mathscr{A}$ has FS. By Theorem (3.2) we have $p \approx \sum_{i=1}^{n} p_{i}^{\prime} \otimes e_{i i}$, where $\left\{p_{i}^{\prime}\right\}$ is a set of projections in $\mathscr{A}$. The remaining work is to adjust $\left\{p_{i}^{\prime}\right\}$. We use induction on $n$.

If $n=2, p \approx p_{1}^{\prime} \otimes e_{11}+p_{2}^{\prime} \otimes e_{22}$, where $p_{1}^{\prime}$ and $p_{2}^{\prime}$ are projections in $\mathscr{A}$. Combining Lemma (2.1) and Lemma (2.2), we obtain that $p_{1}^{\prime} \approx q_{1}+q_{2}$ in $\mathscr{A}$, where $q_{1}$ and $q_{2}$ are two projections in $\mathscr{A}$ such that $q_{1} \leq p_{2}^{\prime}$ and $q_{2} \leq 1-p_{2}^{\prime}$. It follows that $p \approx\left(q_{1}+q_{2}\right) \otimes e_{11}+p_{2}^{\prime} \otimes$ $e_{22}$. Working in the hereditary $C^{*}$-subalgebra of $M_{n}(\mathscr{A})$ generated by $\left(1-q_{1}\right) \otimes e_{11}+1 \otimes e_{22}$, we have $q_{2} \otimes e_{11}+p_{2}^{\prime} \otimes e_{22} \approx\left(p_{2}^{\prime}+q_{2}\right) \otimes e_{22}$ by Lemma (3.1). It follows that $p \approx q_{1} \otimes e_{11}+\left(p_{2}^{\prime}+q_{2}\right) \otimes e_{22}$. Let $p_{1}=q_{1}$ and $p_{2}=q_{2}+p_{2}^{\prime}$.

Assume that $p \approx \sum_{i=1}^{n} p_{i}^{\prime} \otimes e_{i i}$ such that $p_{2}^{\prime} \leq p_{3}^{\prime} \leq \cdots \leq p_{n}^{\prime}$. Applying Lemma (2.1) and Lemma (2.2) to $p_{1}^{\prime}$, and $p_{n}^{\prime}$, we have $p_{1}^{\prime} \approx q_{n}+q_{n}^{\prime}$, where $q_{n}$ and $q_{n}^{\prime}$ are projections in $\mathscr{A}$ such that $q_{n} \leq$ $1-p_{n}^{\prime}$ and $q_{n}^{\prime} \leq p_{n}^{\prime}$. By the same argument as in the last paragraph 
we have that $p \approx q_{n}^{\prime} \otimes e_{11}+\sum_{i=2}^{n-1} p_{i}^{\prime} \otimes e_{i i}+\left(p_{n}^{\prime}+q_{n}\right) \otimes e_{n n}$. Repeating this argument to $q_{n}^{\prime}$ and $p_{n-1}^{\prime}$, we have that $q_{n}^{\prime} \approx q_{n-1}^{\prime}+q_{n-1}$, where $q_{n-1}^{\prime}$ and $q_{n-1}$ are two projections in $\mathscr{A}$ such that $q_{n-1} \leq p_{n}^{\prime}-p_{n-1}^{\prime}$ and $q_{n-1}^{\prime} \leq p_{n-1}^{\prime}$. It follows that $p \approx q_{n-1}^{\prime} \otimes e_{11}+\sum_{i=2}^{n-2} p_{i}^{\prime} \otimes e_{i i}+$ $\left(p_{n-1}^{\prime}+q_{n-1}\right) \otimes e_{n-1, n-1}+\left(p_{n}^{\prime}+q_{n}\right) \otimes e_{n n}$.

Proceeding in this way, we write $p_{1}^{\prime}=\sum_{i=1}^{n} q_{i}$, where $\left\{q_{i}\right\}$ is a set of mutually orthogonal projections in $\mathscr{A}$ such that $q_{i} \leq p_{i+1}^{\prime}-p_{l}^{\prime}$ for $2 \leq$ $i \leq n$ (where $\left.p_{n+1}^{\prime}=1\right), q_{1} \leq p_{2}^{\prime}$, and $p \approx q_{1} \otimes e_{11}+\sum_{i=2}^{n}\left(p_{i}^{\prime}+q_{i}\right) \otimes e_{i i}$. Let $p_{1}=q_{1}$ and $p_{i}=p_{i}^{\prime}+q_{i}$ for $2 \leq i \leq n$. Then $p_{1} \leq p_{2} \leq \cdots \leq p_{n}$ and $p \approx \sum_{i=1}^{n} p_{i} \otimes e_{i i}$.

M. A. Rieffel raised a question in [18, 7]: If $\mathscr{A}$ is a unital $C^{*}$ algebra with cancellation, and if two projections $p$ and $q$ in $M_{n}(\mathscr{A})$ represent the same class in $K_{0}(\mathscr{A})$, are $p$ and $q$ in the same path component of projections in $M_{n}(\mathscr{A})$ ? Since $\mathscr{A}$ has cancellation, $[p]=[q]$ in $K_{0}(\mathscr{A})$ if and only if $p \sim q$ ([3] or [4]). Hence, Rieffel's question is equivalent to whether two Murray-von Neumann equivalent projections in $M_{n}(\mathscr{A})$ are in the same path component of projections in $M_{n}(\mathscr{A})$. The following corollary provides a partial answer for his question in the case that $\mathscr{A}$ has FS:

3.4. Corollary. If $\mathscr{A}$ is a unital $C^{*}$-algebra with FS and cancellation, and if $p$ and $q$ are two projections in $M_{n}(\mathscr{A})$, then $p \sim q$ if and only if $p \approx q$.

Proof. Of course we need only to show that $p \sim q$ implies $p \approx$ $q$. Since $M_{n}(\mathscr{A})$ has FS, by Theorem (3.2) we have $p \approx q_{1}+q_{2}$, where $q_{1}$ is a subprojection of $q$ and $q_{2}$ is a subprojection of $1-q$. Since $\mathscr{A}$ has cancellation and $p \sim q, q_{2} \sim q-q_{1}$. Working in $\left(1-q_{1}\right) M_{n}(\mathscr{A})\left(1-q_{1}\right)$, by Lemma (3.1) we can find a unitary $v$ in $U_{0}\left(\left(1-q_{1}\right) M_{n}(\mathscr{A})\left(1-q_{1}\right)\right)$ such that $v q_{2} v^{*}=q-q_{1}$. Set $u=q_{1}+v$. Then $u$ is a unitary in $U_{0}\left(M_{n}(\mathscr{A})\right)$ such that $u q_{1}=q_{1} u$. Thus $p \approx q_{1}+q_{2} \approx q$.

Concerning the unitary orbit of elements in $M_{n}(\mathscr{A})$, we have the following corollary:

3.5. COROLLARY. If $\mathscr{A}$ is a $C^{*}$-algebra with FS and $x$ is a normal element in $M_{n}(\mathscr{A})$ with finite spectrum, then there is a unitary element $u$ in $U_{n}^{0}(\mathscr{A})$ such that $u x u^{*}=\sum_{j=1}^{n}\left[\sum_{i=1}^{n} \lambda_{i} p_{i j}\right] \otimes e_{j j}$, where $\left\{p_{i j}\right\}$ is a set of projections in $\mathscr{A}$ such that $p_{i_{1} j} \perp p_{i_{2} j}$ in $\mathscr{A} \otimes e_{j j}$ if $i_{1} \neq i_{2}$. 
Proof. By operator calculus we write $x=\sum_{i=1}^{m} \lambda_{i} p_{i}$, where $\left\{\lambda_{i}\right\}$ is a set of complex numbers and $\left\{p_{i}\right\}$ is a set of mutually orthogonal projections in $M_{n}(\mathscr{A})$. By Theorem (3.2) we can find a unitary element $u_{1}$ in $U_{n}^{0}(\mathscr{A})$ such that $u_{1} p_{1} u_{1}^{*}=\sum_{j=1}^{n} p_{1 j} \otimes e_{j j}\left(=q_{1}\right)$ for some projections $\left\{p_{1 j}\right\}$ in $\mathscr{A}$. Working in $\left(I_{n}-q_{1}\right) M_{n}(\mathscr{A})\left(I_{n}-q_{1}\right)$ and repeating the same argument, we can find a unitary $u_{2}^{\prime}$ in $U_{0}\left[\left(I_{n}-q_{1}\right) M_{n}(\mathscr{A})\left(I_{n}-q_{1}\right)\right]$ such that $u_{2}^{\prime}\left(u_{1} p_{2} u_{1}^{*}\right) u_{2}^{\prime 2}=\sum_{j=1}^{n} p_{2 j} \otimes e_{j j}$ for some projections $\left\{p_{2 j}\right\}$ in $\mathscr{A}$. It follows from $p_{1} p_{2}=0$ that $p_{1 j} p_{2 l}=0$ for $1 \leq j<l \leq n$. Set $u_{2}=q_{1}+u_{2}^{\prime}$. Then $u_{2}$ is a unitary in $U_{n}^{0}(\mathscr{A})$ and $u_{2} u_{1}\left(p_{1}+p_{2}\right) u_{1}^{*} u_{2}^{*}=\sum_{i=1}^{2} \sum_{j=1}^{n} p_{i j} \otimes e_{j j}=$ $\sum_{j=1}^{n}\left(\sum_{i=1}^{2} p_{i j}\right) \otimes e_{j j}$.

Proceeding in this way we can find unitary elements $\left\{u_{i}: 1 \leq i \leq\right.$ $m\}$ in $U_{n}^{0}(\mathscr{A})$ such that

$$
\begin{array}{r}
u_{m} u_{m-1} \cdots u_{1}\left(p_{1}+p_{2}+\cdots+p_{m}\right) u_{1}^{*} \cdots u_{m-1}^{*} u_{m}^{*} \\
=\sum_{i=1}^{m}\left[\sum_{j=1}^{n} p_{i j} \otimes e_{j j}\right]=\sum_{j=1}^{n}\left[\sum_{i=1}^{m} p_{i j}\right] \otimes e_{j j} .
\end{array}
$$

Let $u=u_{m} \cdots u_{2} u_{1}$. It is obvious that $u$ is in $U_{n}^{0}(\mathscr{A})$ and

$$
u x u^{*}=\sum_{j=1}^{n}\left[\sum_{i=1}^{m} \lambda_{l} p_{i j}\right] \otimes e_{j j} .
$$

It is well known that the unitary orbit of a self-adjoint matrix in $M_{n}(\mathbb{C})$ contains a diagonal self-adjoint matrix. If $\mathbb{C}$ is replaced by a unital $C^{*}$-algebra with FS, we have the following weaker analogue:

3.6. Corollary. If $\mathscr{A}$ is a $C^{*}$-algebra with FS and $x$ is a selfadjoint element in $M_{n}(\mathscr{A})(n \geq 1)$, then for any $\varepsilon>0$ there exist a unitary element $u$ in $U_{n}^{0}(\mathscr{A})$ and elements $a_{i}$ in $\mathscr{A}$ with finite spectra such that

$$
\left\|u x u^{*}-\sum_{i=1}^{n} a_{i} \otimes e_{i i}\right\|<\varepsilon .
$$

Proof. Since $M_{n}(\mathscr{A})$ has FS, there is a self-adjoint element $h$ in $M_{n}(\mathscr{A})$ with finite spectrum such that $\|x-h\|<\varepsilon$. By the same argument as in the proof of Corollary (3.5) we can find a unitary element $u$ in $U_{n}^{0}(\mathscr{A})$ such that $u h u^{*}=\sum_{l=1}^{n} a_{i} \otimes e_{i i}$, where $\left\{a_{i}\right\}$ is a set of self-adjoint elements in $\mathscr{A}$ with finite spectra. Therefore,

$$
\left\|u x u^{*}-\sum_{i=1}^{n} a_{i} \otimes e_{i i}\right\|=\|x-h\|<\varepsilon .
$$


3.7. REMARK. Concerning the computation of $K_{0}$-groups of a $C^{*}$ algebra, M. A. Rieffel raised a question in [18, 8]: What is the smallest $n$ such that the projections in $M_{n}(\mathscr{A})$ generate $K_{0}(\mathscr{A})$ ? Theorem (3.3) provides a partial answer for his question for the class of $C^{*}$ algebras with FS (actually it has been given in [22] although it was not mentioned there). In fact, if $\mathscr{A}$ is a $C^{*}$-algebra with FS, then the smallest such an integer is $n=1$; in other words, $K_{0}(\mathscr{A})$ is generated by the set of Murray-von Neumann equivalence classes of projections in $\mathscr{A}$.

\section{Diagonalizing projections in $M(\mathscr{A})$.}

4.1. Theorem. Assume that $\mathscr{A}$ is a $\sigma$-unital $C^{*}$-algebra with FS and $\left\{e_{n}\right\}$ is a fixed increasing sequential approximate identity consisting of projections. If $p$ is a projection in $M(\mathscr{A})$, then the following hold:

(i) There is a unitary $u$ in $M(\mathscr{A})$ connected to the identity by a path of unitaries, where the path is continuous in the strict topology, such that $u p u^{*}=\sum_{i=1}^{\infty} p_{i}$, where $p_{i} \leq e_{i}$ for $i \geq 1$; in other words, each strict path component of projections in $M(\mathscr{A})$ contains a diagonal projection with respect to $\left\{e_{n}\right\}$.

(ii) There exist a unitary $v$ in $U_{0}(M(\mathscr{A}))$ and a subsequence $\left\{e_{m_{i}}\right\}$ of $\left\{e_{n}\right\}$ such that $v p v^{*}=\sum_{i=1}^{\infty} p_{i}^{\prime}$, where $p_{i}^{\prime}$ is a projection of $\left(e_{m_{i}}-e_{m_{i-1}}\right) \mathscr{A}\left(e_{m_{i}}-e_{m_{i-1}}\right)$ for $i \geq 1$; in other words, each norm path component of projections in $M(\mathscr{A})$ contains a block-diagonal projection with respect to $\left\{e_{n}\right\}$.

Before proving this theorem, we state the following corollary, which can be regarded as an analogue of the well known fact that a projection on a separable Hilbert space is unitarily equivalent to a diagonal projection whose diagonal entries are either 1 or 0 .

4.2. Corollary. If $\mathscr{A}$ is a $\sigma$-unital $C^{*}$-algebra with FS, and if $p$ is a projection in $L\left(\mathscr{H}_{\mathscr{A}}\right)$, then there is a unitary $u$ in $L\left(\mathscr{H}_{\mathscr{A}}\right)$ such that $u p u^{*}=\sum_{i=1}^{\infty} p_{i} \otimes e_{i i}$, where $\left\{p_{i}\right\}$ is a sequence of projections in $\mathscr{A}$. Consequently, $p \approx \sum_{i=1}^{\infty} p_{i} \otimes e_{i i}($ by [8]).

Proof of Theorem (4.1).

Case 1. If $p$ is a projection of $\mathscr{A}$.

Choose $n \geq 1$ large enough such that $\left\|p\left(1-e_{n}\right) p\right\|$ is small. Then Lemma (2.1) of [10] applies. We find a unitary $u$ in $U_{0}(M(\mathscr{A}))$ such 
that $u p u^{*} \leq e_{n}$. By Theorem (3.2), $p \approx u p u^{*} \approx \sum_{i=1}^{n} p_{i}$, where $p_{i} \leq e_{i}-e_{i-1}$ for $1 \leq i \leq n$. Hence both (i) and (ii) hold.

Case 2. If $p$ is a projection in $M(\mathscr{A}) \backslash \mathscr{A}$.

Let $\left\{q_{n}\right\}$ and $\left\{q_{n}^{\prime}\right\}$ be two increasing sequences of projections in $\mathscr{A}$ such that $q_{n} \nearrow p$ and $q_{n}^{\prime} \nearrow 1-p$ in the strict topology. Set $f_{n}=q_{n}+q_{n}^{\prime}$. Then $\left\{f_{n}\right\}$ is an increasing sequential approximate identity of $\mathscr{A}$ consisting of projections. By the argument of $[10,2.4]$ we find a unitary element $v$ in $U_{0}(M(\mathscr{A}))$ such that

$$
e_{m_{1}} \leq v f_{n_{1}} v^{*} \leq e_{m_{2}} \leq v f_{n_{2}} v^{*} \leq e_{m_{3}} \leq \cdots,
$$

where $\left\{n_{i}\right\}$ and $\left\{m_{i}\right\}$ are increasing sequences. It is clear that

$$
v p v^{*}=\sum_{i=1}^{\infty} v p\left(f_{n_{i}}-f_{n_{t-1}}\right) v^{*}=\sum_{i=1}^{\infty} v\left(q_{n_{t}}-q_{n_{t-1}}\right) v^{*}
$$

and $v\left(q_{n_{t}}-q_{n_{t-1}}\right) v^{*} \leq v\left(f_{n_{t}}-f_{n_{i-1}}\right) v^{*}=\left(v f_{n_{l}} v^{*}-e_{m_{l}}\right)+\left(e_{m_{t}}-v f_{n_{t-1}} v^{*}\right)$ (where $q_{n_{0}}=0$ and $f_{n_{0}}=0$ ).

We first prove (i). By Theorem (3.2) we find a unitary $w_{i}$ in $U_{0}\left(\mathscr{A}_{i}\right)$, where $\mathscr{A}_{i}=\left[v\left(f_{n_{l}}-f_{n_{l-1}}\right) v^{*}\right] \mathscr{A}\left[v\left(f_{n_{l}}-f_{n_{l-1}}\right) v^{*}\right]$, such that $w_{i} v\left(q_{n_{t}}-q_{n_{t-1}}\right) v^{*} w_{i}^{*}=r_{i}+r_{i}^{\prime}$, where $r_{i} \leq v f_{n_{t}} v^{*}-e_{m_{t}}$ and $r_{i}^{\prime} \leq$ $e_{m_{t}}-v f_{n_{t-1}} v^{*}$. Set $w=\sum_{i=1}^{\infty} w_{i}$. Then $w$ is a unitary in $M(\mathscr{A})$ such that $w$ is path connected (in the strict topology) to the identity and

$$
w v p v^{*} w^{*}=\sum_{i=1}^{\infty}\left(r_{i}+r_{i}^{\prime}\right) \leq \sum_{i=1}^{\infty}\left[\left(v f_{n_{i}} v^{*}-e_{m_{i}}\right)+\left(e_{m_{i}}-v f_{n_{i-1}} v^{*}\right)\right] .
$$

Since $r_{i}+r_{i+1}^{\prime} \leq e_{m_{i+1}}-e_{m_{l}}$, we can apply Theorem (3.2) again to get a unitary $w_{i}^{\prime}$ in $U_{0}\left(\mathscr{B}_{i}\right)$, where $\mathscr{B}_{i}=\left(e_{m_{t+1}}-e_{m_{i}}\right) M(\mathscr{A})\left(e_{m_{t+1}}-e_{m_{t}}\right)$ such that

$$
w_{i}^{\prime}\left(r_{i}+r_{i+1}^{\prime}\right) w_{i}^{\prime *}=\sum_{j=m_{l}+1}^{m_{t+1}} p_{j}
$$

where $p_{j}$ is in $\left(e_{j}-e_{j-1}\right) \mathscr{A}\left(e_{j}-e_{j-1}\right)$ for $m_{i}<j \leq m_{i+1}$.

Define $w^{\prime}=\sum_{i=1}^{\infty} w_{i}^{\prime}$. Then $w^{\prime}$ is a unitary in $M(\mathscr{A})$ such that $w^{\prime}$ is path connected in the strict topology to the identity and $w^{\prime} w v p v^{*} w^{*} w^{\prime *}=\sum_{i=1}^{\infty} p_{i}$. Set $u=w^{\prime} w v$, as (i) desired.

To prove (ii), we start with $p \approx v p v^{*}=\sum_{i=1}^{\infty} v\left(q_{n_{t}}-q_{n_{t-1}}\right) v^{*}$, where $s_{i}=v\left(q_{n_{t}}-q_{n_{t-1}}\right) v^{*} \leq v\left(f_{n_{t}}-f_{n_{t-1}}\right) v^{*}=\left(v f_{n_{t}} v^{*}-e_{m_{t}}\right)+\left(e_{m_{t}}-v f_{n_{i-1}} v^{*}\right)$ for each $1 \geq 1$ and $q_{n_{0}}=0$ and $f_{n_{0}}=0$. With respect to

$$
v\left(f_{n_{l}}-f_{n_{t-1}}\right) v^{*}=\left(v f_{n_{t}} v^{*}-e_{m_{l}}\right)+\left(e_{m_{i}}-v f_{n_{t-1}} v^{*}\right),
$$


we can write

By Lemma (2.3),

$$
s_{i}=\left(\begin{array}{ll}
a_{i} & b_{i} \\
b_{i}^{*} & c_{i}
\end{array}\right) \quad \text { for } i \geq 1
$$

$$
v p v^{*} \approx \sum_{i=1}^{\infty}\left(s_{i}+s_{i}^{\prime}\right)
$$

where $s_{i}$ is a projection in $\left(v f_{n_{i}} v^{*}-e_{m_{i}}\right) \mathscr{A}\left(v f_{n_{i}} v^{*}-e_{m_{l}}\right)$ and $s_{i}^{\prime}$ is a projection in $\left(e_{m_{t}}-v f_{n_{t-1}} v^{*}\right) \mathscr{A}\left(e_{m_{t}}-v f_{n_{t-1}} v^{*}\right)$. Let $p_{i}^{\prime}=s_{i}^{\prime}+s_{i-1}$ for $i \geq 1$, where $s_{0}=0$, as desired.

The following theorem asserts that the unitary orbit of each selfadjoint element of $M(\mathscr{A})$ contains an "almost" diagonal form, which is a natural analogue of the classical Weyl-von Neumann theorem.

4.3. THEOREM. Assume that $\mathscr{A}$ is a $\sigma$-unital $C^{*}$-algebra with FS and also $M(\mathscr{A})$ has FS. If $\left\{e_{n}\right\}$ is a fixed increasing approximate identity of $\mathscr{A}$ consisting of projections and $h$ is a self-adjoint element in $M(\mathscr{A})$, then there exist a unitary $u$ in $M(\mathscr{A})$, an element $a$ in $\mathscr{A}$, some mutually orthogonal subprojection $p_{i j}\left(1 \leq j \leq n_{i}\right)$ of $e_{i}-e_{i-1}$ for each $i \geq 1$ and a real bounded scalar sequence $\left\{\lambda_{i j}\right\}$ such that

$$
\sum_{i j} p_{i j}=1, \quad \text { and } u h u^{*}=\sum_{i=1}^{\infty}\left[\sum_{j=1}^{l_{i}} \lambda_{i j} p_{i j}\right]+a
$$

where a can be chosen such that $\|a\|$ is arbitrarily small. Moreover, $u$ is connected to the identity by a path of unitaries in $M(\mathscr{A})$, where the path is continuous in the strict topology.

4.4. COROLlaRY. If $\mathscr{A}$ is a unital $C^{*}$-algebra with FS and $L\left(\mathscr{H}_{\mathscr{A}}\right)$ has FS also, then for any self-adjoint element $h$ in $L\left(\mathscr{H}_{\mathscr{A}}\right)$ there are a unitary $u$ in $L\left(\mathscr{H}_{\mathscr{A}}\right)$, an element a in $K\left(\mathscr{H}_{\mathscr{A}}\right)$, a sequence of projections $\left\{p_{i j}\right\}$ in $\mathscr{A}$ and a real bounded scalar sequence $\left\{\lambda_{i j}\right\}$ such that

$$
\sum_{i=1}^{\infty}\left(\sum_{j=1}^{l_{t}} p_{i j}\right) \otimes e_{i i}=1 \text { and } u h u^{*}=\sum_{i=1}^{\infty}\left[\sum_{j=1}^{l_{t}} \lambda_{i j} p_{i j}\right] \otimes e_{i i}+a,
$$

where $p_{i j}\left(i \leq j \leq l_{i}\right)$ are mutually orthogonal for each fixed $i$, and a can be chosen with an arbitrarily small norm.

Proof of Theorem (4.3).. Since $\mathscr{A}$ is $\sigma$-unital and both $\mathscr{A}$ and $M(\mathscr{A})$ have FS, by [21, 3.1] we can find mutually orthogonal projections $p_{i}$ in $\mathscr{A}$ with $\sum_{i=1}^{\infty} p_{i}=1$, a real bounded scalar sequence 
$\left\{\lambda_{i}\right\}$ and an element $b$ in $\mathscr{A}$ with arbitrarily small norm such that $h=\sum_{i=1}^{\infty} \lambda_{i} p_{i}+b$. Let $f_{n}=\sum_{i=1}^{n} p_{i}$. Then $\left\{f_{n}\right\}$ is an increasing approximate identity consisting of projections. By the same argument as in $[10,2.4]$ we can find a unitary $v$ in $M(\mathscr{A})$ such that $v \sim 1$, and

$$
e_{m_{1}} \leq v f_{n_{1}} v^{*} \leq e_{m_{2}} \leq v f_{n_{2}} v^{*} \leq e_{m_{3}} \leq \cdots,
$$

where $\left\{n_{i}\right\}$ and $\left\{m_{i}\right\}$ are increasing sequences. Since

$$
v\left(\sum_{j=n_{t-1}+1}^{n_{i}} p_{i}\right) v^{*}=\left(v f_{n_{t}} V^{*}-e_{m_{\imath}}\right)+\left(e_{m_{l}}-V f_{n_{l-1}} v^{*}\right)
$$

(where $f_{n_{0}}=0$ ), by the same arguments in the proof of Theorem (4.1) we can find a unitary $w_{i}$ of $\left[v\left(f_{n_{i}}-f_{n_{i-1}}\right) v^{*}\right] M(\mathscr{A})\left[v\left(f_{n_{t}}-f_{n_{t-1}}\right) v^{*}\right]$ path connected to the identity $v\left(f_{n_{i}}-f_{n_{i-1}}\right) v^{*}$ such that

$$
w_{i} v\left(\sum_{j=n_{i-1}+1}^{n_{t}} p_{i}\right) v^{*} w_{i}^{*}=\sum_{j=n_{t-1}+1}^{n_{t}} w_{i} v p_{i}^{\prime} v^{*} w_{i}^{*}+\sum_{j=n_{t-1}+1}^{n_{t}} w_{i} v p_{i}^{\prime \prime} v^{*} w_{i}^{*},
$$

where

$$
\begin{aligned}
p_{i}^{\prime}+p_{i}^{\prime \prime}=p_{i}, \quad r_{i} & =\sum_{j=n_{t-1}+1}^{n_{t}} w_{i} v p_{i}^{\prime} v^{*} w_{i}^{*}=v f_{n_{i}} v^{*}-e_{m_{\imath}} \quad \text { and } \\
r_{i}^{\prime} & =\sum_{j=n_{t-1}+1}^{n_{t}} w_{i} v p_{i}^{\prime \prime} v^{*} w_{i}^{*}=e_{m_{t}}-v f_{n_{t-1}} v^{*}
\end{aligned}
$$

Let $w=\sum_{i=1}^{\infty} w_{i}$. Then $w$ is a unitary in $M(\mathscr{A})$ such that $w$ is connected to the identity by a path of unitaries, where the path is continuous in the strict topology. Since $r_{j}+r_{j+1}^{\prime} \leq e_{m_{J+1}}-e_{m_{j}}$, by the same arguments in the proof of Theorem (4.1), we obtain a unitary $w_{j}^{\prime}$ of $\left(e_{m_{J+1}}-e_{m_{J}}\right) M(\mathscr{A})\left(e_{m_{J+1}}-e_{m_{j}}\right)$ path connected to the identity $e_{m_{j+1}}-e_{m}$, such that

$$
w_{j}^{\prime}\left(r_{j}+r_{j+1}^{\prime}\right) w_{j}^{\prime *}=\sum_{i=m_{j}+1}^{m_{j+1}} \sum_{j=1}^{l_{l}} p_{i j},
$$

where $\left\{p_{i j}: 1 \leq j \leq l_{i}\right\}$ is a set of mutually orthogonal subprojections in $\left(e_{i}-e_{i-1}\right) \mathscr{A}\left(e_{i}-e_{i-1}\right)$.

Define $w^{\prime}=\sum_{i=1}^{\infty} w_{i}^{\prime}$. Then $w^{\prime}$ is a unitary in $M(\mathscr{A})$ such that $w^{\prime}$ is path connected to the identity, where the path is continuous in the strict topology. Set $u=w^{\prime} w v$. Then $u$ is path connected to the 
identity, where the path is continuous in the strict topology. It is easily verified that $u h u^{*}$ has a desired form. (Notice that $\left\{\lambda_{i}\right\}$ is equal to $\left\{\lambda_{i j}\right\}$ as sets.)

4.5. Remarks. (i) The condition " $M(\mathscr{A})$ has FS" in the hypotheses of Theorem (4.3) and Corollary (4.4) has been studied in [5], [21] and [24]. Actually many multiplier algebras have the FS property.

(ii) Several applications of the results in this note have been given in the author's subsequent papers [24, Part II, III, IV].

Acknowledgment. The author wishes to thank the referee for pointing out an inaccuracy in Theorem (4.1) in the primary manuscript of this paper.

\section{REFERENCES}

[1] C. A. Akemann, G. K. Pedersen and J. Tomiyama, Multipliers of $C^{*}$-algebras, J. Funct. Anal., 13 (1973), 277-301.

[2] J. Bunce and J. Deddens, $A$ family of simple $C^{*}$-algebras related to weighted shift operators, J. Funct. Anal., 19 (1975), 13-24.

[3] B. Blackadar, Notes on the structure of projections in simple $C^{*}$-algebras, Semesterbericht Funktionalanalysis, W82, Tübingen, March 1983.

[4] _ , $K$-Theory for Operator Algebras, Springer-Verlag, New York, Berlin, Heidelberg, London, Paris, Tokyo, 1987.

[5] L. G. Brown and G. K. Pedersen, $C^{*}$-algebras of real rank zero, preprint, 1989.

[6] L. G. Brown, Extensions of AF algebras: the projection lifting problem, Operator Algebras and Applications, Proc. Symp. Pure Math., 38 (1981), Part I, 175-176.

[7] R. Busby, Double centralizers and extensions of $C^{*}$-algebras, Trans. Amer. Math. Soc., 132 (1968), 79-99.

[8] J. Cuntz and N. Higson, Kuiper's theorem for Hilbert modules, Operator Alg. and Math. Phys., Vol. 62, Proc. of a Summer Conference June 17-21, 1985.

[9] M.-D. Choi and G. A. Elliott, Density of the self-adjoint elements with finite spectrum in an irrational rotation $C^{*}$-algebra, Math. Scand., (to appear).

[10] G. A. Elliott, Derivations of matroid $C^{*}$-algebras II, Ann. of Math., 100 (1974), 407-422.

[11] K. Grove and G. K. Pedersen, Diagonalizing matrices over $C(X)$, J. Funct. Anal., 59 (1984), 65-89.

[12] P. R. Halmos, A Hilbert Space Problem Book, second edition, Springer-Verlag New York, Heidelberg, Berlin, 1982.

[13] R. V. Kadison, Diagonalizing matrices over operator algebras, Bull. Amer. Math. Soc., 8 (1983), 84-86.

[14] __ Diagonalizing matrices, Amer. J. Math., 106 (1984), 1451-1468.

[15] G. Kasparov, Hilbert $C^{*}$-modules: Theorems of Stinespring and Voiculescu, J. Operator Theory, 4 (1980), 133-150.

[16] G. K. Pedersen, The linear span of projections in simple $C^{*}$-algebras, J. Operator Theory, 4 (1980), 289-296.

[17] M. A. Reiffel, The homotopy groups of the unitary groups of non-commutative tori, J. Operator Theory, 17 (1987), 237-254. 
[18] M. A. Reiffel, Non-stable K-theory and non-commutative tori, Contemporary Math., 62 (1987), 267-279.

[19] _ Dimension and stable rank in the $K$-theory of $C^{*}$-algebras, Proc. London Math. Soc., 46 (1983), 301-333.

[20] H. Weyl, Uber beschrankte quadratischen formen deren differentz vollstetig ist, Rend. Circ. Mat. Palermo, 27 (1909), 373-392.

[21] S. Zhang, $K_{1}$-groups, quasidiagonality and interpolation by multiplier projections, Trans. Amer. Math. Soc., to appear.

[22] _ A Riesz decomposition property and ideal structure of multiplier algebras, J. Operator Theory, (to appear).

[23] _ On the structure of projections and ideals of corona algebras, Canad. J. Math., 41 (1989), 721-742.

[24],$C^{*}$-algebras with real rank zero and the internal structure of their corona and multiplier algebras, Part I, II, IV, preprints. Part III to appear in Canad. J. Math.

[25] _ A property of purely infinite simple $C^{*}$-algebras, Proc. Amer. Math. Soc., to appear.

Received November 1, 1988 and in revised form September 28, 1989.

UNIVERSITY OF KANSAS

LAWRENCE, KS 66045-2142 


\section{PACIFIC JOURNAL OF MATHEMATICS EDITORS}

\author{
V. S. VARAdarajaN \\ (Managing Editor) \\ University of California \\ Los Angeles, CA 90024-1555-05 \\ Herbert Clemens \\ University of Utah \\ Salt Lake City, UT 84112 \\ Thomas ENRIGHT \\ University of California, San Diego \\ La Jolla, CA 92093
}

R. FINN

Stanford University

Stanford, CA 94305

HermanN FlaschKa

University of Arizona

Tucson, AZ 85721

VAUGHan F. R. Jones

University of California

Berkeley, CA 94720

STEVEN KeRCKHOFF

Stanford University

Stanford, CA 94305
C. C. Moore University of California Berkeley, CA 94720

MARTIN SCHARLEMANN University of California Santa Barbara, CA 93106

\section{HAROLD STARK}

University of California, San Diego La Jolla, CA 92093

\section{ASSOCIATE EDITORS}
R. ARENS
E. F. BECKeNBACH
B. H. NeumanN
F. WolF
(1904-1989)
K. Yoshida (1906-1982)

\section{SUPPORTING INSTITUTIONS}

UNIVERSITY OF ARIZONA

UNIVERSITY OF BRITISH COLUMBIA

CALIFORNIA INSTITUTE OF TECHNOLOGY

UNIVERSITY OF CALIFORNIA

MONTANA STATE UNIVERSITY

UNIVERSITY OF NEVADA, RENO

NEW MEXICO STATE UNIVERSITY

OREGON STATE UNIVERSITY
UNIVERSITY OF OREGON

UNIVERSITY OF SOUTHERN CALIFORNIA

STANFORD UNIVERSITY

UNIVERSITY OF HAWAII

UNIVERSITY OF TOKYO

UNIVERSITY OF UTAH

WASHINGTON STATE UNIVERSITY

UNIVERSITY OF WASHINGTON 


\section{Pacific Journal of Mathematics}

Vol. 145, No. 1 September, 1990

Sheldon Jay Axler and Allen Lowell Shields, Extensions of harmonic and

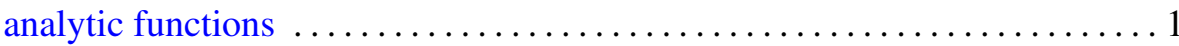

Labib Haddad and Yves Sureau, Les cogroupes et la construction de

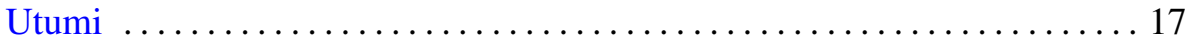

John Hutchinson, Poincaré-Sobolev and related inequalities for submanifolds of $\mathbf{R}^{N}$

Yuk Jaum Leung and Glenn E. Schober, Some coefficient problems and

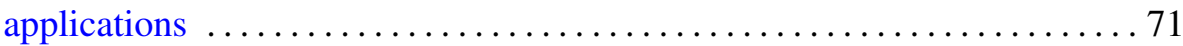

Daniel Ruberman, Seifert surfaces of knots in $S^{4} \ldots \ldots \ldots \ldots \ldots \ldots \ldots \ldots$

Joel Harold Shapiro and Carl Sundberg, Isolation amongst the

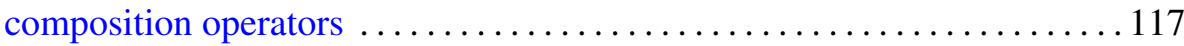

Hans Wenzl, Representations of braid groups and the quantum Yang-Baxter

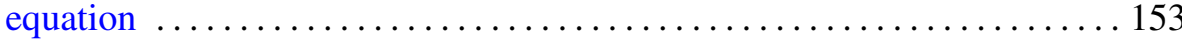

Shuang Zhang, Diagonalizing projections in multiplier algebras and in matrices over a $C^{*}$-algebra 